This is the peer reviewed version of the following article: Amintowlieh, Y., Tzoganakis, C., \& Penlidis, A. (2016). An Overview of the Potential of UV Modification of Polypropylene. Macromolecular Symposia, 360(1), 96-107, which has been published in final form at https://doi.org/10.1002/masy.201500110. This article may be used for non-commercial purposes in accordance with Wiley Terms and Conditions for Self-Archiving.

\title{
An Overview of the Potential of UV Modification of Polypropylene
}

\author{
Yasaman Amintowlieh, Costas Tzoganakis, and Alexander Penlidis*
}

Institute for Polymer Research, University of Waterloo, 200 University Avenue

West, Waterloo, Ontario N2L 3G1, Canada; E-mail: penlidis@uwaterloo.ca

\begin{abstract}
Summary: UV radiation with a photoinitiator has been utilized to modify polypropylene (PP). Factors affecting solid state photomodification were studied and the optimal processing conditions for formation of three different structures (degraded, long chain branched and crosslinked) in PP were determined. A long radiation time (more than 5 minutes) is required to form long chain branched PP (LCBPP) and this exposure time is even higher for production of crosslinked PP (XPP). Trimethylolpropane triacrylate (TMPTA) was utilized to decrease UV radiation duration. Optimal concentrations of TMPTA and photoinitiator for formation of long chain branched and crosslinked PP were determined. Finally, a setup was suggested for continuous photomodification of PP using a twin screw extruder. In this design, PP strands are irradiated directly after the die. Optimal photoinitiator (benzophenone) concentration, coagent (TMPTA) concentration and radiation time required to form controlled rheology (CRPP) or long chain branched PP or crosslinked PP were identified.
\end{abstract}

Keywords: polypropylene, UV radiation, long chain branching, crosslinking, rheology

\section{Introduction}

\subsection{Background}

Polypropylene (PP) is one of the most common thermoplastics with numerous applications ranging from household appliances to automotive interior, due to its high stiffness and resistance to environmental stress cracking and solvents. Each application requires a specific PP grade with a specific molecular structure, molecular weight (MW) and molecular weight distribution (MWD). PP 
molecular weight and polydispersity index (PDI) affect the melt flow behaviour, processing characteristics and, eventually, the final application of PP. Due to different applications and production processes of PP, it is important to find a versatile technique to modify its MW and MWD, or to form special structures to fit specific application and post-polymerization processes.

A commercial way to synthesize PP with specific stereo-regularity is by using Ziegler-Natta (ZN) catalysts. The molecular weight of PP which is produced by this method is in the range of $3 \mathrm{x}$ $10^{5}$ to $7 \times 10^{5}$. The corresponding PDI is within the range of 5 to 20 for heterogeneous and 2 to 5 for homogenous Z-N catalysts. ${ }^{[1]}$ Industries synthesizing PP are mainly utilizing heterogeneous Ziegler-Natta catalysts. ${ }^{[2]}$ Control over MWD of the PP which is produced by this synthesis method is difficult. ${ }^{[1]}$ Since 1990 , metallocenes have been used for producing isotactic PP. PP produced by a metallocene catalyst has a narrower molecular weight distribution. ${ }^{[3]}$ Metallocene catalysis has not only been utilized for controlling the MWD, but also for producing grades of PP with long chain branches in their backbone (long chain branched PP). ${ }^{[4,5]}$

Despite developments made for synthesizing PP with tailored structures by metallocene catalysts, post-synthesis modification methods (also known as post-reactor modification techniques) still remain more popular since they are cost-effective, have straightforward steps and require regular equipment for PP modification. Moreover, metallocene catalysis did not have the same success as with polyethylene and the statistics show that in 2010 only 2 to $3 \%$ of the world's polypropylene demand was provided from metallocene catalysis. ${ }^{[6]}$

The post-reactor modification of PP has been used to form three general grades of polypropylene: Controlled Rheology, Long Chain Branched and Crosslinked PP. The molecular structure, property and processing application for each grade are briefly explained below. 


\section{Controlled Rheology PP (CRPP)}

This grade of modified PP has an increased melt flow rate (MFR) via degradation. The molecular weight is lower and the molecular weight distribution is (usually) narrower than the parent PP. This grade of PP is utilized in injection molding and can lead to faster processing of the polymer.

\section{Long Chain Branched PP (LCBPP)}

In spite of the large market of polypropylene, poor rheological properties, such as lack of melt strength, limit its processing in certain applications. Thus, by modifying the rheology and enhancing strain hardening of the PP melt, PP can be processed via different methods, such as foaming, thermoforming, extrusion coating and blow molding. ${ }^{[7-9]}$ The high melt strength of a polymer is

either due to long chain branching (LCB) or high molecular weight. ${ }^{[10]}$ Formation of long chain branches (LCBs) in the PP structure is found to be the most efficient method to increase its melt strength (or, equivalently, its extensional viscosity).

Crosslinked Polypropylene (XPP)

Polypropylene has superior mechanical properties compared to polyethylene. However, its applications are limited in fields like the structural and wire coating industry. In these applications crosslinked polyethylene is commonly used due to its high heat, stress crack and solvent resistance and good mechanical properties. The limited usage of PP in these applications, despite better heat resistance, higher melting point and mechanical properties, is due to the inefficiency of its crosslinking process. 
In post-reactor modification, the idea is to start with a common commercial PP which is synthesized via Z-N catalysis, modify this parent PP and prepare three main grades of PP, as described above.

Several methods have been introduced in the literature for post-reactor modification of PP, either through controlled degradation or introduction of LCBs and/or crosslinks. Methods presented in the literature for hydrogen abstraction from the PP backbone can be divided into two categories: methods using thermo-chemical initiators and methods using high energy radiation. The use of peroxides that can be activated by heat falls into the former, while gamma and electron beam (EB) radiation belong to the latter category. Photoinitiators can also be used for macroradical generation and they can be activated using UV energy. This method can also fall into the second category, as high energy UV radiation causes excitation of the photoinitiator and subsequent hydrogen abstraction. In this overview, the UV photoinitiation process for PP post-reactor modification is explained and results presented in recent publications on different types of PP modification are reviewed. Moreover, optimal conditions for formation of any of these structures in PP via photomodification are identified.

\subsection{UV Modification Mechanisms}

For modification with UV radiation, photoinitiators such as benzophenone (BPH) have been used. Photoinitiators are activated by UV radiation rather than thermal energy and are less toxic than peroxides. Controlling photoinitiation is easier than peroxide thermal initiation, since mixing temperature and residence time can be adjusted without activating the initiator (unlike peroxides which are decomposed by increasing temperature). Thus, efficient mixing of PP and initiator can be reached prior to initiating the reaction. BPH activation via $\mathrm{UV}$ radiation is shown in Table 1 (reaction a). 
$\mathrm{BPH}$ absorbs light in the wavelengths of 215, 254 and $330 \mathrm{~nm}$ and becomes excited. At the excited state, it can abstract a tertiary hydrogen from the PP backbone and form a macroradical (reaction b in Table 1). Since PP tertiary macroradicals are unstable, $\beta$-scission reactions are highly probable. As shown in reaction c of Table $1, \beta$-scission results in a smaller macroradical and a chain with a double bond at its end.

$\beta$-scission reactions play an important role in formation of any of the molecular structures mentioned earlier. As mentioned above, as a result of $\beta$-scission, macroradicals and chains with a double bond at their end will form. If this is followed by disproportionation termination of macroradicals (reaction d in Table 1), controlled rheology PP is expected.

In order to form long chain branched structures, radicals should be formed first and $\beta$-scission reactions are responsible for formation of macroradicals through degradation. It has been found that formation of branches and crosslinks are only evident after degradation of PP due to $\beta$-scission. ${ }^{[11,12]}$ Thus, in the beginning of radiation, $\beta$-scission is dominant; however, after the concentration of macroradicals reaches a certain limit, bimolecular combination will become more probable (reaction e in Table 1).

If photomodification is carried out beyond this step (long chain branching), the probability of hydrogen abstraction from PP chains which are already long chain branched increases. In this case, interconnected networks will form (reaction $\mathrm{f}$ in Table 1), which leads to production of crosslinked PP (XPP).

Formation of any of these structures via photomodification requires specific operational conditions which lead to certain reactions from Table 1 becoming dominant. Thus, it is important to identify significant operational variables, their effects on the molecular structure of PP, and the optimal 
conditions (optimal window) for formation of any of the three main molecular structures (CRPP, LCBPP and XPP) discussed above.

\section{Experimental Section}

In the studies considered in this overview, two different grades of PP homopolymer with the same MFR (3.5g/10 min) were utilized. For the first study presented (batch modification, sections 3.1 and 3.2), Pro-fax PH 382M from LyondellBasell was used; in the second part (the effect of coagent on PP photomodification and continuous photomodification of PP, sections 3.3 and 3.4) PP2255E1 from ExxonMobil was used. Trimethylolpropane triacrylate (TMPTA) was purchased from Sigma-Aldrich and used as coagent. Irganox 1010, Ciba Specialty Chemicals, was used as antioxidant to prevent PP thermal degradation.

In the batch photomodification processes, PP pellets along with photoinitiator were melt-mixed in a batch mixer at 190 0C and $100 \mathrm{rpm}$ for 8 minutes. After grinding, PP/initiator blended granules were compression-moulded into discs with $25 \mathrm{~mm}$ diameter and $1 \mathrm{~mm}$ thickness at $190{ }^{\circ} \mathrm{C}$ under an applied force of 4,400 $\mathrm{N}$ for five minutes. The discs were subsequently irradiated using a mercury UV lamp (Versa Cure). For the continuous photomodification part, the radiation setup is explained in detail separately (see section 3.4).

A stress-controlled parallel plate rheometer (AR2000, TA instruments) was used to measure the rheological properties of the irradiated samples at $190^{\circ} \mathrm{C}$. Parallel plates with diameter of $25 \mathrm{~mm}$ were used for all shear tests. Strain sweeps were carried out to identify the linear viscoelastic region during the tests. Frequency sweeps were subsequently performed in the range of $0.01-100 \mathrm{~Hz}$. From these tests, storage modulus ( $\left.G^{\prime}\right)$, loss modulus $\left(G^{\prime \prime}\right)$, complex modulus $\left(G^{*}\right)$, loss tangent $(\tan \delta)$, and complex viscosity $\left(\eta^{*}\right)$ were obtained at different angular frequencies $(\omega)$. 
Table 1: Reactions during PP photomodification with benzophenone

ID

a

b
Chemical Reaction

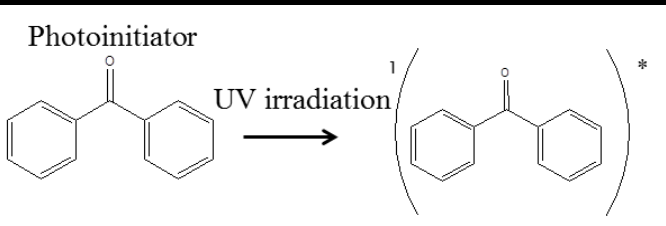

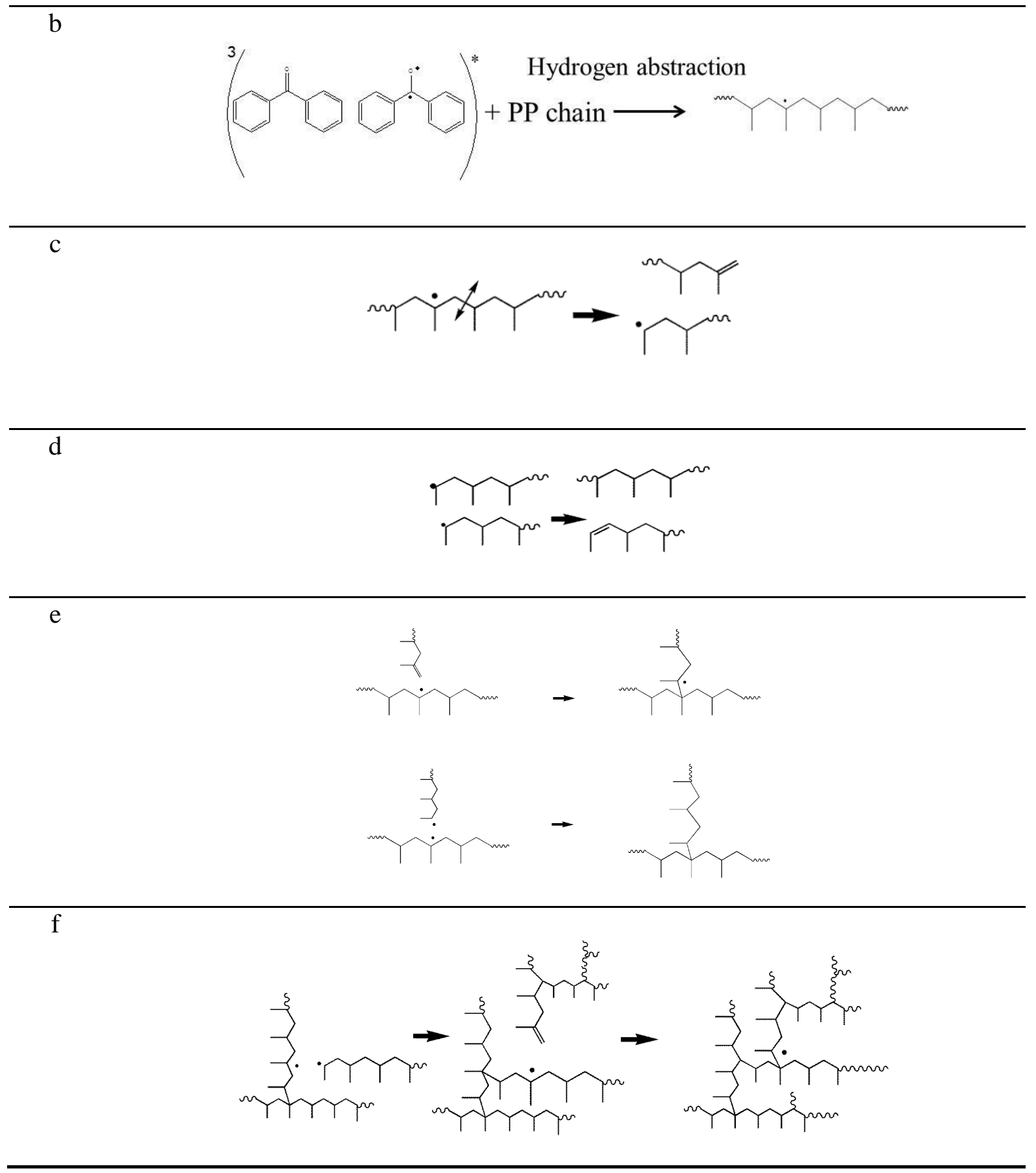

7 
The Cross model (Equation (1)) was fit on $\eta^{*}$ vs. $\omega$ data to determine the shear thinning index (n), zero shear viscosity $\left(\eta_{0}\right)$ and relaxation time $(\lambda)$ of each sample.

$$
\eta^{*}=\frac{\eta_{0}}{1+(\omega \lambda)^{n}}
$$

In Equation (1), $\eta_{0}$ is the limiting value of the complex viscosity at very low shear rates where the viscosity of the polymer melt is independent of shear rate (Newtonian viscosity). $\lambda$ represents the terminal relaxation time whose inverse is related to a characteristic shear rate for departure from the Newtonian plateau. $\mathrm{n}$ is the shear thinning index, which is the slope of the shear thinning region in the $\eta^{*}-\omega$ plots. An increase in the zero shear viscosity $\left(\eta_{0}\right)$ of a sample compared to the parent PP indicates higher molecular weight, which can be due to the presence of long chain branches. Long chain branched PP (LCBPP) has larger zero shear viscosity $\left(\eta_{0}\right)$ and relaxation time $(\lambda)$, but smaller shear thinning index (n) than linear PP. ${ }^{[13]}$

Rheological polydispersity indices are determined using Equation (2) to (4). ${ }^{[14]}$

$$
\begin{gathered}
P I=\frac{10^{5}}{G_{c}(\mathrm{~Pa})} \\
\text { ModSep }=\frac{\omega^{\prime}}{\omega^{\prime \prime}}
\end{gathered}
$$




$$
\mathrm{ER}=\mathrm{C}_{1} G^{\prime} \text { at } G^{\prime \prime}=500 \mathrm{~Pa}
$$

PI is the so-called rheological "polydispersity index", ModSep stands for "Modulus Separation" and ER is another polydispersity index, more indicative of the high molecular weight end, as in-

troduced by Shroff and Mavridis. ${ }^{[14,15]}$ These useful rheological polydispersity indices relate to MWD breadth and branching. Gc in Equation (2) is the crossover modulus, which is the modulus at which $G^{\prime}$ and $G^{\prime \prime}$ are equal. In Equation (3), $\omega^{\prime}$ and $\omega^{\prime \prime}$ are the angular frequencies when $G^{\prime}$ and $\mathrm{G}^{\prime \prime}$ are equal to $1000 \mathrm{~Pa}$. In Equation (4) $\mathrm{C}_{1}$ is the slope of the $\log \left(\mathrm{G}^{\prime}\right)$ versus $\log \left(\mathrm{G}^{\prime \prime}\right)$ curve.

In Equation (2) and Equation (3), PI correlates inversely with Gc, and ModSep shows the G' and G" distance from each other at a specific modulus $(1000 \mathrm{~Pa})$. It is expected that the values of PI and ModSep reflect the MWD of the resins, while ER is only sensitive to the high molecular weight (MW) end of the MWD (essentially, it is the polydispersity of the high molecular weight chains). [14]

The percent gel content of selected samples was found by extraction. The method followed is described in ASTM D2765-11.

\section{Results and Discussion: PP Photomodification}

\subsection{Batch Modification}

In this section, the significant factors for PP molecular structure modification are considered first. The operational windows for formation of CRPP, LCBPP and XPP are presented next. In order to study the effect of operational conditions on formation of different structures in PP, processing variables such as radiation time, UV lamp intensity and cooling rate, along with concentration (and 
type) of photoinitiator, were manipulated. It was found that all variables and most two factor interactions significantly affected the molecular structures of the photomodified PP. The operating conditions that result in PP with narrower MWD and lower MW (CRPP), long chain branched PP with improved strain hardening behavior and crosslinked PP were identified.

In order to study the processing variables and identify optimal processing conditions, a D-optimal design of experiments was carried out. The above mentioned variables were studied within the ranges explained in reference [16]. Table 2 (copied from reference [16]) shows these variables and their limits within the design of experiments.

Table 2: Photomodification operational variables affecting PP molecular structure

\begin{tabular}{ccc}
\hline Factor & Process variable (units) & Ranges \\
\hline A & Photoinitiator concentration (wt \%) & $0.1-0.5$ (with respect to polymer mixture) \\
\hline B & Duration of radiation (s) & $120-600$ \\
\hline C & UV lamp intensity (\%) & $47-100$ (with respect to total lamp intensity) \\
\hline D & Cooling air pressure (\%) & $0-100$ (with respect to total air flow pres- \\
& & sure) \\
\hline E & Type of photoinitiator & BPH and DEBPH \\
\hline
\end{tabular}

Two different photoinitiators (benzophenone $(\mathrm{BPH})$ and 4,4' bis-diethylamino-benzophenone $(\mathrm{DEBPH}))$ were employed and their effects on PP modification were studied along with other variables (photointiator concentration, radiation time, lamp intensity, duration of UV exposure). By analyzing viscoelastic properties, empirical models were acquired for each viscoelastic response $\left(\eta_{0}, \lambda, n\right.$, ER, ModSep and PI). See reference [16] for more details on the effect of the variables on viscoelastic properties and PP molecular structure. 
In order to find conditions that maximize LCB while having the lowest gel content in CRPP, contour plots were constructed for $\eta_{0}, \lambda, n, E R$ and gel content. Certain specifications were set for each response in order to locate the (optimal) processing window. Table 3 shows these specifications that lead to optimal processing conditions for both LCBPP and CRPP.

Table 3: Specifications for optimal processing conditions for formation of LCBPP and CRPP

\begin{tabular}{ccc}
\hline Response & Specifications for CRPP & Specifications for LCBPP \\
\hline $\mathrm{n}$ & $>0.58$ & $<0.48$ \\
\hline$\eta_{0}(\mathrm{~Pa} . \mathrm{s})$ & $<7800$ & $>10,000$ \\
\hline$\lambda(\mathrm{s})$ & $<0.67$ & $>3$ \\
\hline $\mathrm{ER}$ & $<1.19$ & $>0.4$ \\
\hline Gel content $(\%)$ & 0 & $<5$ \\
\hline
\end{tabular}

Figure 1 shows the operational window corresponding to the specifications of Table 3 for formation of CRPP and LCBPP in the presence of two different photointiators (BPH and DEBPH). Figure 1a suggests that samples with high BPH concentration should be irradiated for long radiation time at low temperature and low lamp intensity to form branches. On the other hand, Figure $1 \mathrm{~b}$ shows formation of long chain branches in PP when DEBPH is used at low concentration (0.25 wt-\%) and both temperature and lamp intensity at the high level.

These trends confirm that different photoinitiators require different conditions to form certain structures in PP. In general, formation of long chain branches in PP was easier and modification was possible over a broader operational window when BPH was used as photoinitiator.

As expected, for formation of controlled rheology PP, either with BPH or DEBPH, high temperature is required. High temperature encourages $\beta$-scission and degradation of PP. ${ }^{[17]}$ Thus, no air 
cooling is required for formation of CRPP. Also, in order to form controlled rheology PP, DEBPH is a more efficient photointiator with a wider operational window. Both Figures $1 \mathrm{~b}$ and $1 \mathrm{~d}$ show that DEBPH is more effective when temperature is higher, since high cooling air pressure (which controls the temperature of radiation) discourages formation of long chain branches and degradation of PP. This might be due to the bulkier structure of DEBPH, which makes movements of the molecule within the solid more difficult. Thus, high temperature assists the movement of the radical centers in solid PP and encourages both degradation and long chain branching when bulkier photoinitiators like DEBPH are used. In Figure 1, zero air pressure means using no cooling air and consequently high radiation temperature $\left(\mathrm{T}>80^{\circ} \mathrm{C}\right)$.

On the other hand, it is known that BPH has slower kinetics and a lower molecular extinction coefficient. Thus, radiation at low temperature for long time (more than 5 minutes) can lead to formation of LCBs (as per the mechanism explained in section 1.2).

In summary, conditions shown in Figure 1a were found to be optimal for LCB structure formation in PP. Thus, these conditions (low lamp intensity, low temperature and high BPH concentration $(0.5 \mathrm{wt}-\%))$ were used to produce LCBPP. The radiation time was increased from 1 to 15 minutes to study the effect of radiation duration in more detail. It was found that by increasing radiation time, LCBs will be formed first. By further increasing the radiation time, crosslinked structures and gel content in the samples increases (mechanism discussed in section 1.2). Figure 2 shows the gel content in PP samples after 5, 10 and 15 minutes radiation. Although formation of crosslinks keeps increasing with radiation time, the rate of crosslinking via photoinitiation is rather low. In section 3.3, a more efficient method for formation of XPP is discussed.

Since long chain branched and crosslinked structures have been produced more effectively when BPH is used as photoinitiator, this photoinitiator was chosen for the rest of this study. 


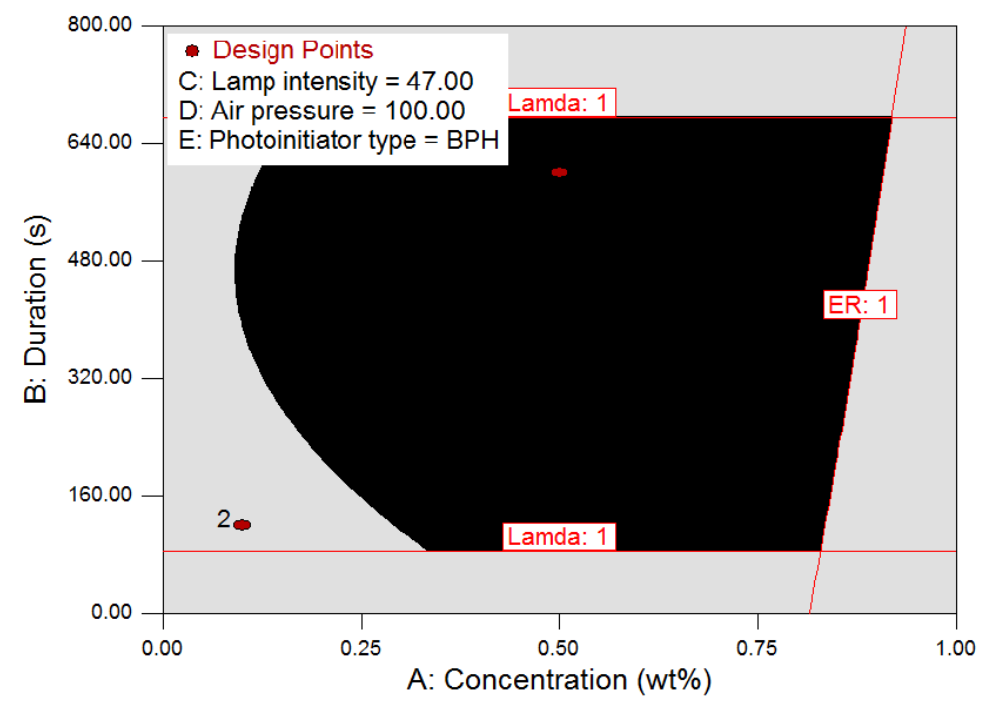

a

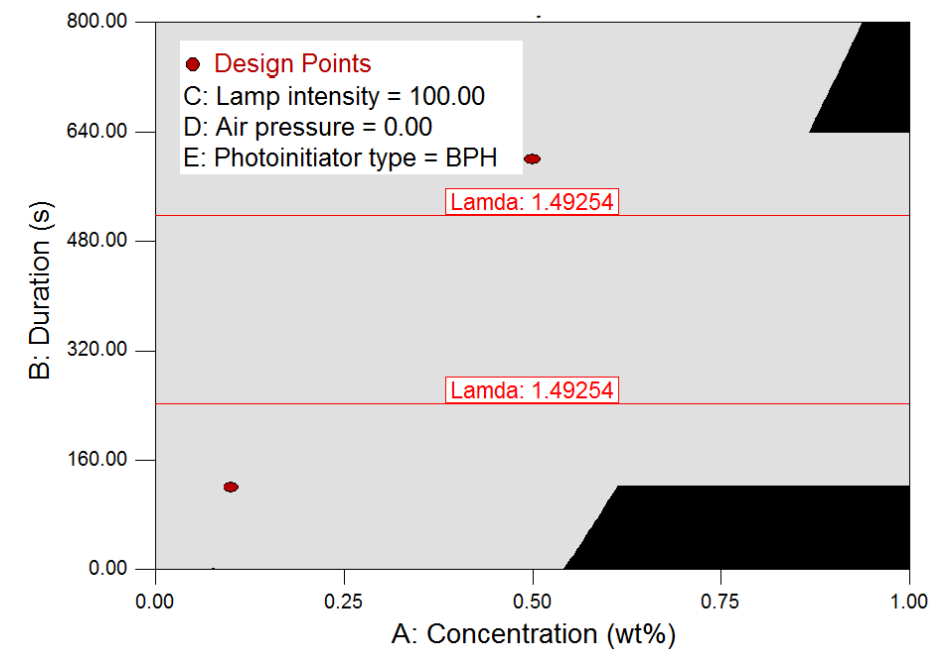

$\mathrm{c}$

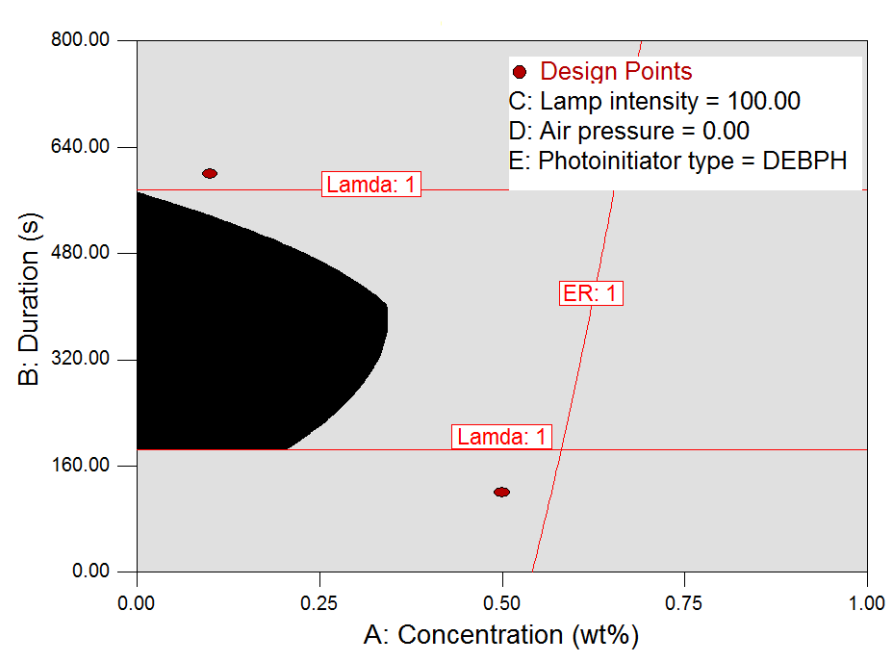

b

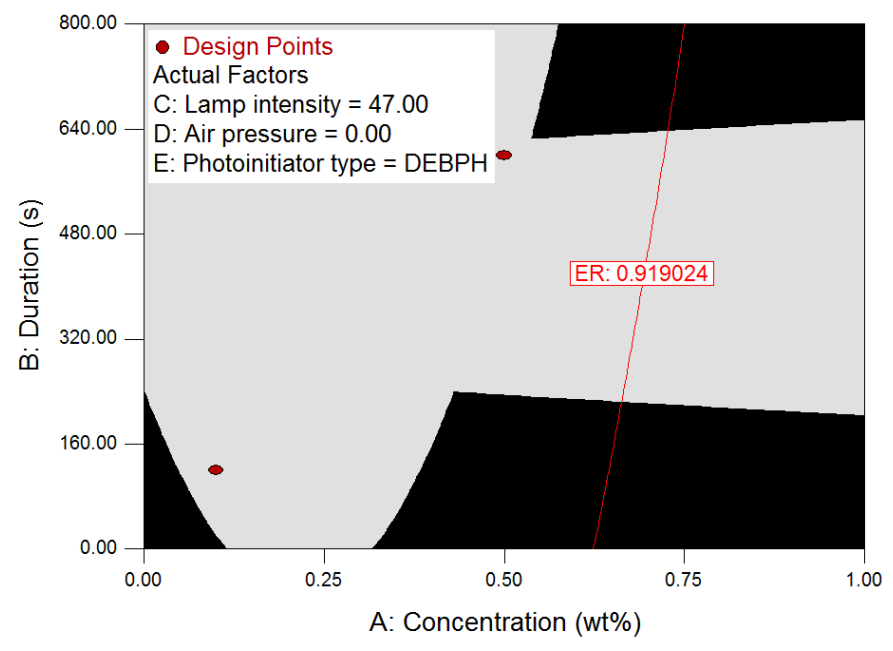

d

Figure 1: Optimal operational conditions for formation of long chain branches using BPH (a) and DEBPH (b); operational window for formation of crosslinked PP using BPH (c) and DEBPH (d) 


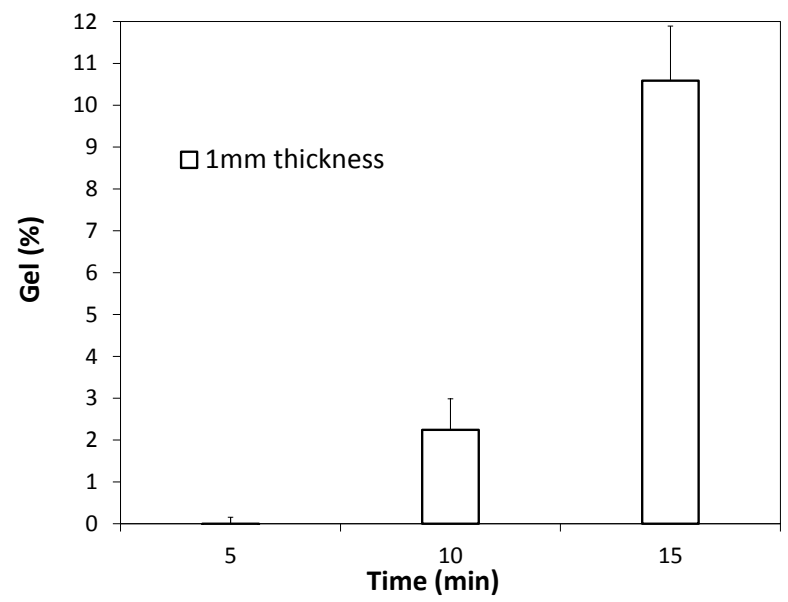

Figure 2: Gel content in the $1 \mathrm{~mm}$ thick samples radiated for 5, 10 and 15 minutes

\subsection{Limitations}

Photomodification can be employed to successfully modify the PP molecular structure. ${ }^{[16]}$ However, there are certain limitations to this technique which should be addressed.

1) Limited UV penetration depth: UV penetrates up to a limited thickness in the sample. This limited penetration depth should be identified for the specific radiation conditions.

2) Long UV exposure time is required for PP photomodification: radiation time significantly affects the molecular structure of the modified PP. Thus, it is important to find the minimum UV exposure time which is required to form significant amounts of LCB. It is worth noting that long radiation time is mainly a limitation for formation of LCBPP and XPP via BPH, since formation of CRPP is still possible by low exposure time to UV (see Figure 1c).

These two variables (radiation penetration depth and radiation time) were studied at constant lamp intensity and radiation temperature, by preparing PP sheets with thickness ranging from 1 to $3 \mathrm{~mm}$ and by radiating the sheets between 5 to 15 minutes. It was found that the limited thickness that UV can penetrate through PP samples is below $1 \mathrm{~mm}$, when BPH was used as photoinitiator at a lamp intensity of $0.67 \mathrm{~W} / \mathrm{m}^{2}$. It was further found that a significant amount of branches will be 
formed in the sample if radiation is carried out for more than 5 minutes (for a thickness of $1 \mathrm{~mm}$ ). At this thickness, gel will be formed in the sample if radiation continues for more than 6 minutes (onset of gelation). Figure 2 shows gel content of the samples at different radiation times. As radiation time increases, gel content of the samples also increases (at $1 \mathrm{~mm}$ thickness). It can be seen that 15 minutes of radiation time lead to formation of only $11 \%$ of gel in PP. This is because of the low rate of long chain branching and crosslinking reactions and bimolecular combination of macromolecules in the solid PP.

In order to address the issue of long radiation time required for the formation of LCBPP and XPP, multifunctional comonomers (coagents) can be utilized.

\subsection{Utilizing Coagent to Decrease Exposure Time to UV}

Coagents are reactive multifunctional monomers that react with the radical center and prevent $\beta$ scission, so the radical center can react with another PP macroradical and form branches. In PP modification via different peroxides, acrylic and allylic coagents have been utilized to form long chain branches. ${ }^{[18-21]}$ Trimethylolpropane triacrylate (TMPTA) is a triacrylic coagent which has been utilized along with peroxides to form LCBPP. It has been found that TMPTA has very fast kinetics and can be successfully utilized to form long chain branched structures in PP at the presence of either photoinitiators or peroxides. Utilizing TMPTA along with photoinitiators can result in formation of significant amount of gel, which makes formation of XPP a straightforward process. ${ }^{[18,22]}$

On the other hand, in order to find conditions that result in a maximum amount of long chain branches with a minimum gel content, variables such as BPH concentration, TMPTA concentration and radiation time have to be manipulated. Figure 3 compares operation conditions for maximum amount of gel (Figure 3a) with the conditions that resulted in maximum amount of long chain 
branches with less than $5 \%$ gel content (Figure 3b). In Figure 3a and 3b, the radiation duration required for formation of branches has significantly decreased after addition of TMPTA (60 s and $35 \mathrm{~s}$ for formation of XPP and LCBPP, respectively).

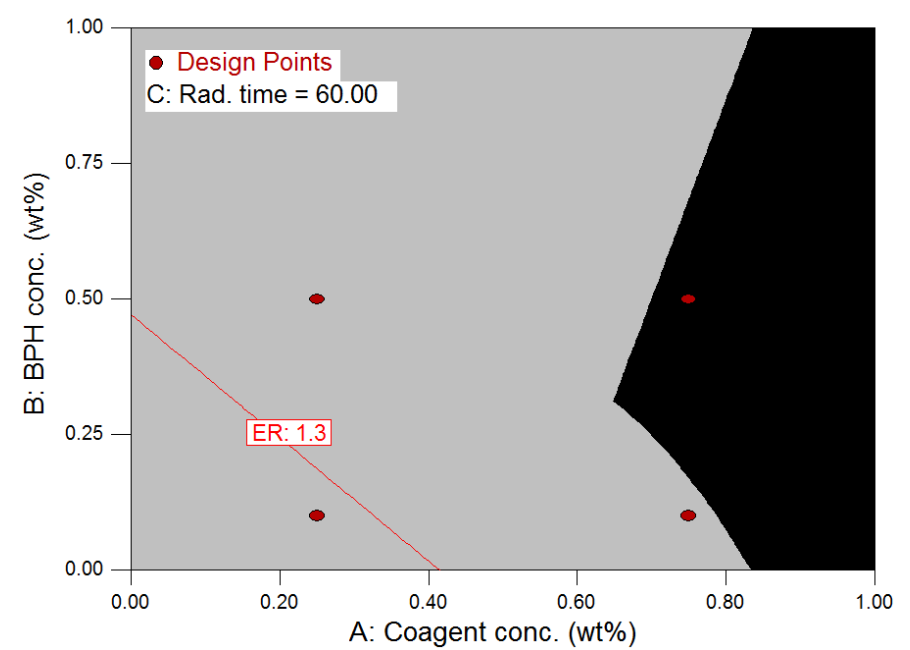

$\mathrm{a}$

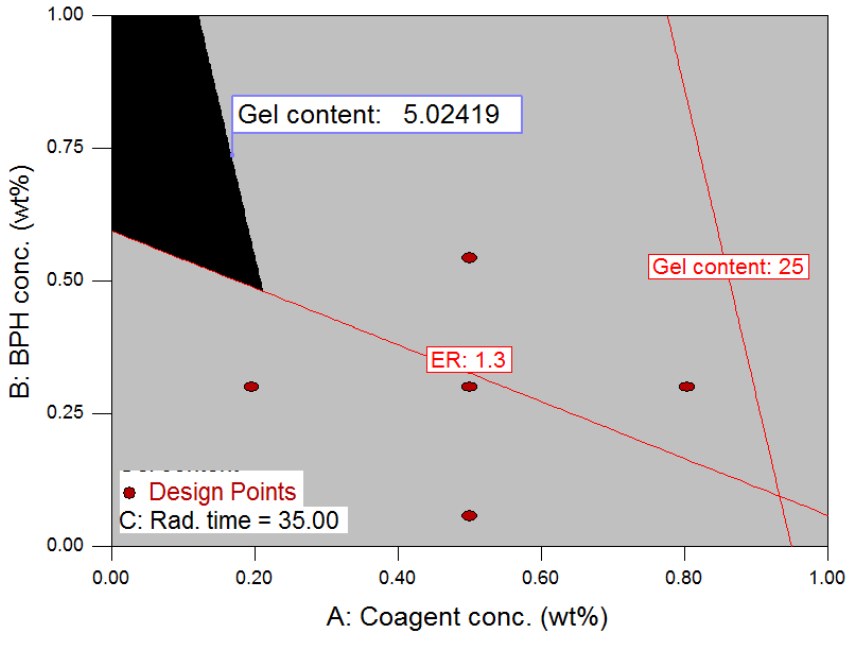

b

Figure 3: Optimal processing conditions for formation of crosslinked (a) and long chain branched (b) PP when BPH (photoinitiator) was used along with TMPTA (coagent)

\subsection{Development of Setup for Continuous Photomodification}

The next step was to scale up the batch photoinitiation to a continuous setup. The continuous photomodification process, same as any reactive extrusion modification, starts with melt mixing PP and photoinitiator in a twin screw extruder.

Prior to this study, reactive extrusion itself had been successfully utilized for PP photomodification. CRPP was produced by mixing benzophenone and polypropylene in the extruder and radiation was carried out in the last two zones of the extruder. By adding coagents such as pentaerythritol triacrylate (PETA) and irradiation inside the extruder, long chain branched PP was produced.

During these reactions, the extruder barrel was removed so the UV lamp could radiate the PP/BPH 
or PP/BPH/coagent melt mixture. ${ }^{[23,24]}$ In these reactions (radiation inside the extruder) formation of long chain branched structures was only possible in the presence of coagents; without the aid of coagents, only degraded PP was formed. ${ }^{[24]}$ The other possible drawback of melt radiation inside the extruder is that UV only penetrates through the surface and this causes inhomogeneities in the modified mixture. ${ }^{[25]}$

In order to avoid the disadvantages of reactive extrusion and radiation of the molten polymer in the extruder, the extrudate was radiated after solidification. In this method, PP and BPH were mixed in a twin screw extruder and radiation was carried out after solidification of this mixture in the cooling bath. Figure 4 shows the schematic for this setup. After the extrudate solidifies in the cooling water bath, it stretches between two free rotating rollers while it is exposed to UV radiation. The exposure time to UV is controlled by the number of times the strand goes back and forth between the rollers and finally the photomodified strand is collected on a winder.

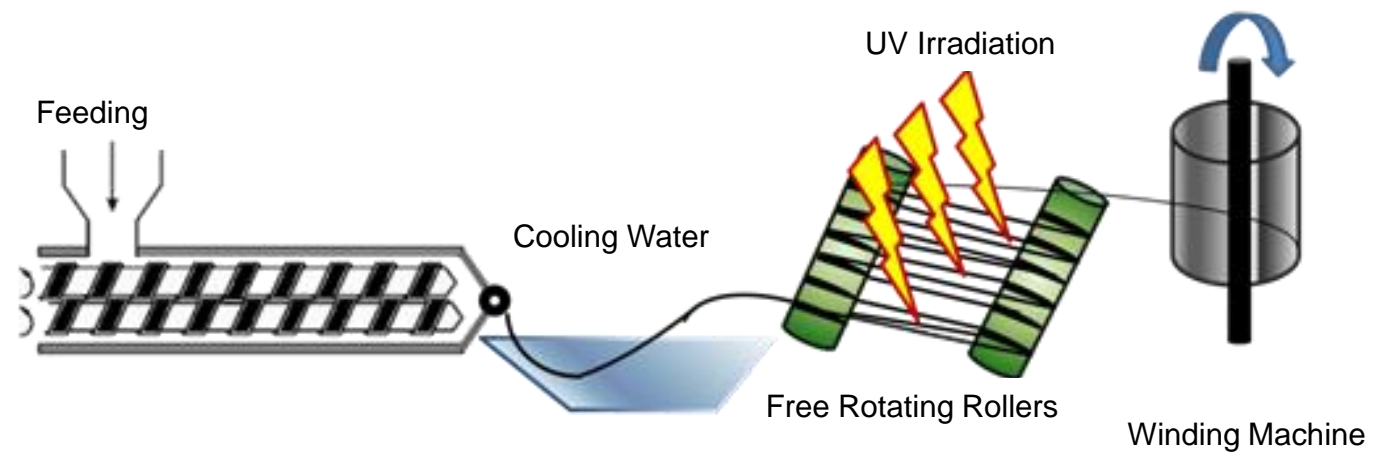

Figure 4: Setup developed for continuous photomodification of polypropylene; copied from reference ${ }^{[25]}$

The extruder throughput rate, die hole diameter and winding machine speed control the thickness of the extrudate strand. Since there is a limited thickness UV can penetrate, it is important to find the optimal thickness for effective radiation through the depth of the samples. Thus, the strands were stretched up to $0.5 \mathrm{~mm}$ prior to radiation. Once again, variables such as BPH concentration, coagent concentration and radiation time were manipulated to identify the operational conditions 
for formation of long chain branched and crosslinked structures. The operating conditions for forming LCBPP and XPP are shown in black in Figure 5a and b, respectively.

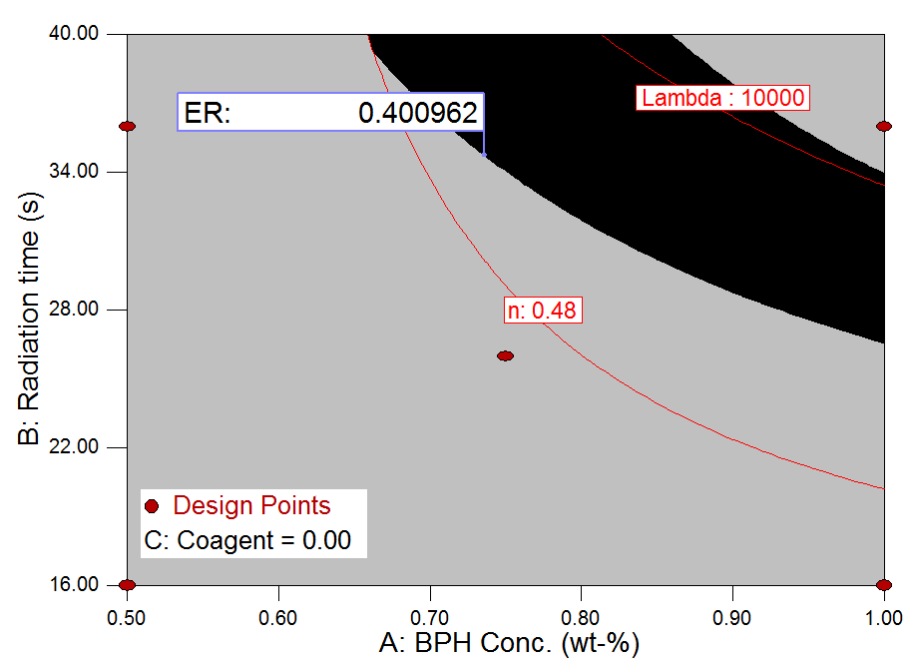

a

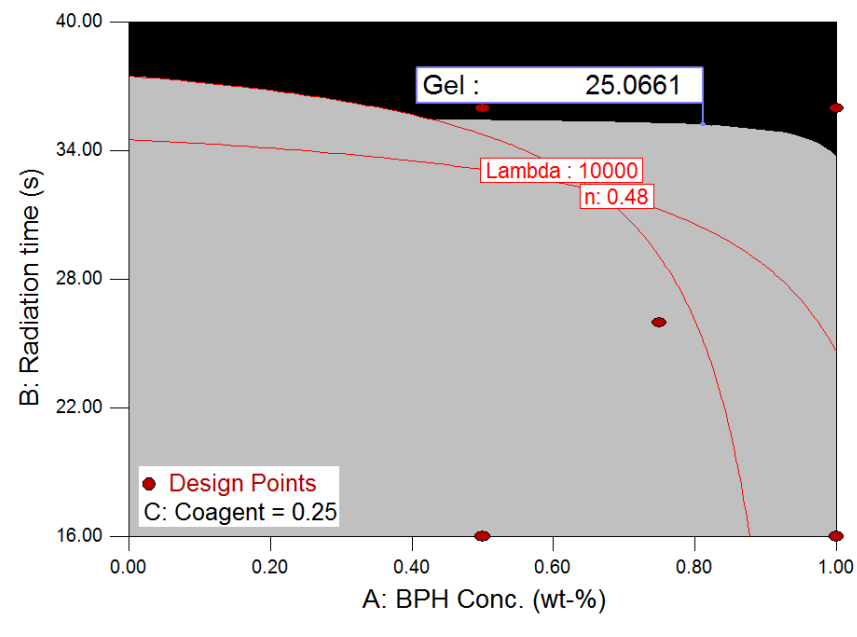

b

Figure 5: Optimal processing conditions for formation of long chain branches (a) and crosslinks (b) under continuous PP photomodification

Figure 5a shows conditions for formation of long chain branches with gel less than 5\%, while Figure $5 \mathrm{~b}$ shows conditions for formation of XPP (more than $25 \%$ gel content). It should be noted that even higher gel content could be reached by longer radiation time and higher TMPTA content. In the continuous photomodification of PP, the radiation time required for formation of LCBPP is significantly lower than that for batch reactions (compare Figure 5a and Figure 1a). This is due to the low thickness of extruded strands which makes UV radiation more efficient. Moreover, postextrusion stretching of the strands limits chain mobility and restricts $\beta$-scission, which is advantageous for formation of LCBPP.

Based on the mechanism shown in Table $1 \mathrm{f}$ for formation of XPP, it is expected that highly crosslinked PP samples have significant amount of LCBs as well as high gel content. In order to study the effect of modification exclusively on the sol fraction of the XPP samples and evaluate the mechanism suggested in Table 1f, sol and gel fractions of XPP were separated via a gel exclusion 
process. In this process, photomodified samples with $25 \%$ gel content were captured within stainless steel pouches (120 mesh) and immersed in boiling xylene for 4 hours. After 4 hours, the pouches containing the insoluble fraction of XPP (gel fraction) were removed from the solution. Then, acetone (non-solvent) was used to precipitate the dissolved polymer. After filtration of the precipitated polymer, residual xylene and acetone were removed by heating the samples at $100{ }^{\circ} \mathrm{C}$ for 12 hours. The remaining polymer, which is the sol fraction of the photomodified XPP, was subsequently used to prepare discs for rheological tests.

Figure 6 shows storage modulus $\left(G^{\prime}\right)$, shear viscosity $\left(\eta^{*}\right)$, and $\tan \delta-G^{*}$ plots of XPP (prepared via photomodification using the conditions shown in Figure 5b) and the parent PP before and after the gel exclusion process (explained above).

Comparison between PP and the photomodified samples before and after gel exclusion shows that the radiated run exhibits a greater G' compared to the parent PP both before and after gel exclusion (Figure 6a). Moreover, even after the gel exclusion process, the photomodified sample has greater $\eta_{0}$ than the parent PP. Lower $\tan \delta$-G* indicates a broader MWD in the radiated run even after gel exclusion (Figure 6c). All the trends in Figure 6 confirm the formation of considerable amount of LCBs in the soluble fraction of XPP. The lower values of G' and $\eta^{*}$ of PP after the gel exclusion procedure are due to degradation after this process. Furthermore, GPC test results also showed that the radiated sample after gel exclusion had about 0.26 branches per polymer molecules (weight average). These GPC results are not shown in this overview (for the sake of brevity) but can be found elsewhere. ${ }^{[25]}$ All these results confirm the mechanism suggested in Table 1f for formation of XPP, and show that XPP samples contain considerable amount of LCBs as well as gelled structures. 


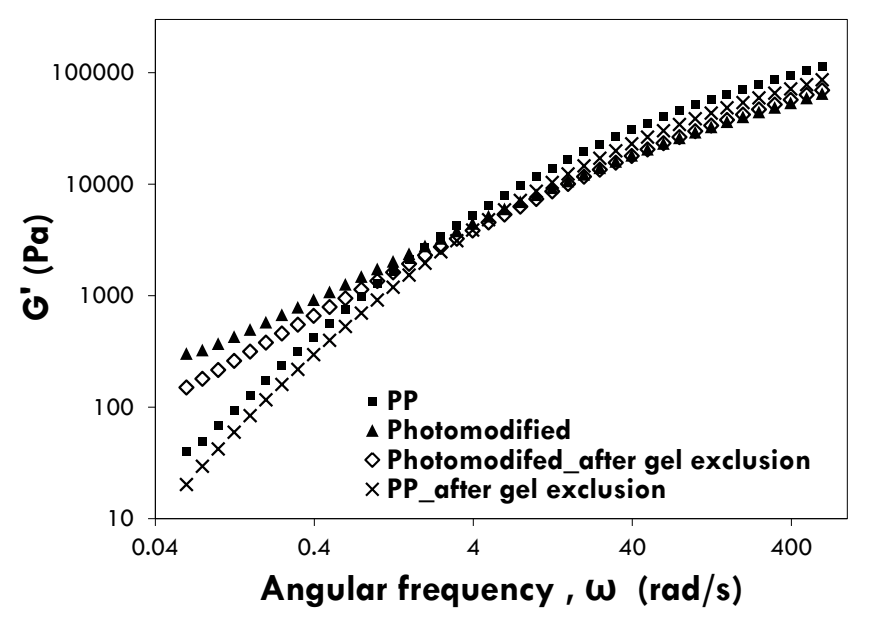

a

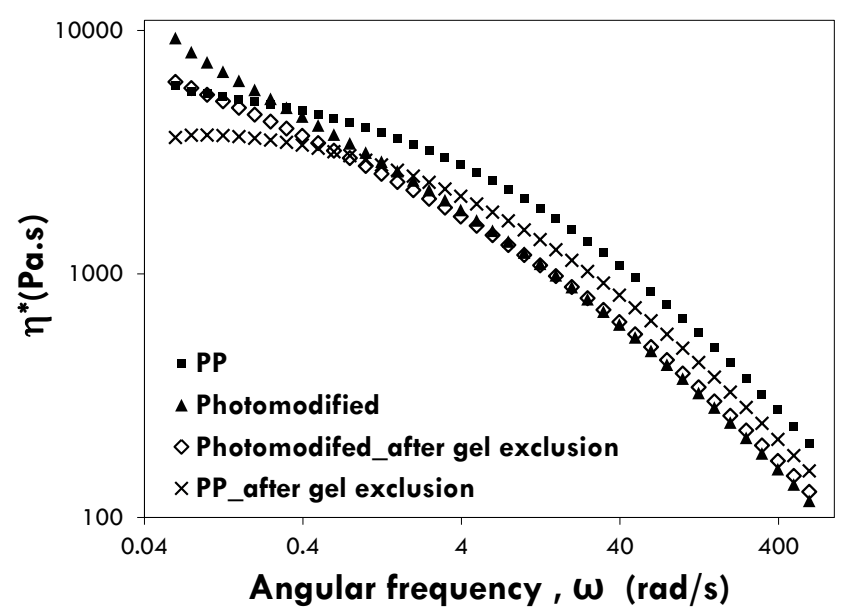

b

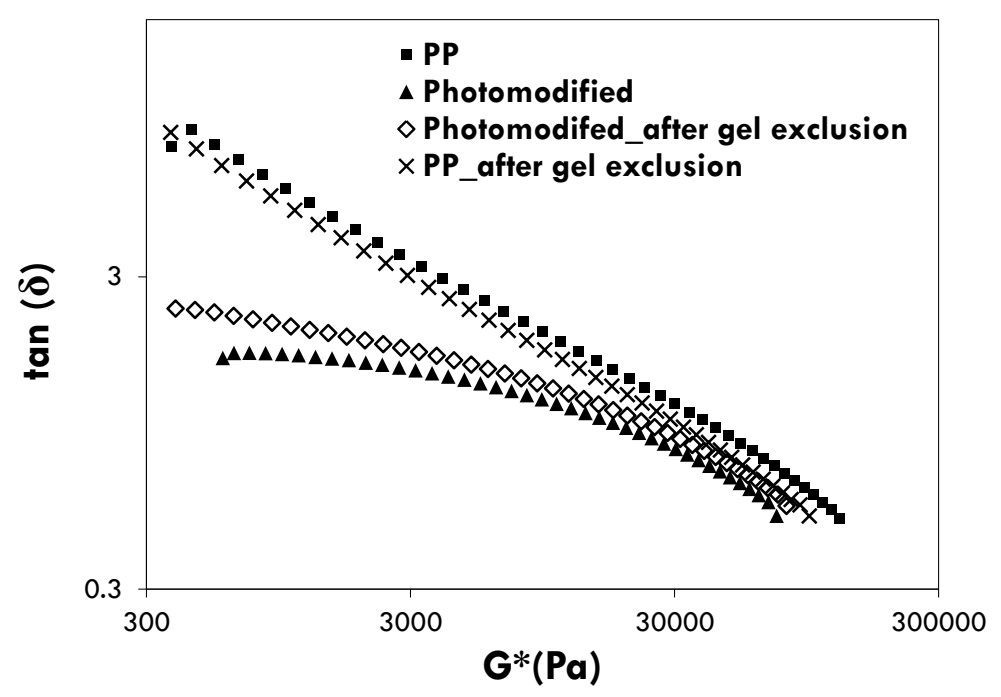

C

Figure 6: $\mathrm{G}^{\prime}-\omega, \eta^{*}-\omega$ and $\tan \delta$-G* for photomodified run and the parent PP before and after gel exclusion

\section{Conclusions}

Post-reactor modification of polypropylene (PP) is important as it aims at changing the molecular structure of PP and synthesizing different grades of PP for different processing applications. These changes include narrowing of the PP molecular weight distribution (MWD) by degradation via $\beta$ scission (controlled rheology PP) or formation of long chain branches (LCBs) in the PP backbone 
or crosslinking of the PP chains. However, $\beta$-scission should be controlled to prevent severe degradation in the polymer. In addition to $\beta$-scission reactions, bimolecular chain combination is necessary to form long chain branched PP (LCBPP). Thus, by adjusting process conditions, PP with different molecular structures can be generated.

In this overview, UV radiation along with a photoinitiator is used to abstract hydrogens from PP backbones and initiate their modification. The final PP molecular structure is affected by photoinitiator concentration, radiation time, UV lamp intensity, radiation temperature and type of photoinitiator used. Different combinations of these variables can result in CRPP, LCBPP or XPP. One of the drawbacks in using UV radiation in polymer modification is its limited penetration depth. In order to investigate the effect of UV penetration depth along with UV radiation time, discs with thickness of 1, 2 and $3 \mathrm{~mm}$ were radiated for 5, 10 and 15 minutes. It was shown that as sample thickness decreased and/or radiation time increased, more LCBs were formed. The limited UV penetration depth in PP solid samples was found to be below $1 \mathrm{~mm}$.

Long radiation time (above $5 \mathrm{~min}$ ), which is necessary to form LCBPP, decreases the potential of this technique for commercialization. Thus, an attempt was made to decrease the required radiation time by using a coagent. Trimethylolpropane triacrylate (TMPTA) was used as a coagent to reduce degradation by stabilizing the radical center. The effects of coagent concentration, BPH concentration and radiation time on the formation of LCBPP and XPP were studied.

Finally, in order to assist in the commercialization and scale up of the PP photomodification, a method was developed to continuously radiate PP. The modification was carried out on solidified strands after extrusion from a twin screw extruder. The strands were stretched and folded several times over two parallel rollers. By manipulating BPH concentration and radiation time, LCBPP and XPP were successfully produced. In general, the results obtained show that UV radiation can 
easily be used to modify PP and form different PP grades, ranging from controlled rheology to long chain branched and crosslinked PP.

Acknowledgements: The authors are grateful for financial support from the Natural Sciences and Engineering Research Council (NSERC) of Canada and the Canada Research (CRC) program.

[1] C. Tzoganakis, J. Vlachopoulos, and A. Hamielec. Polym. Eng. Sci., 1988, 28, 170.

[2] P.C. Mohring and N.J. Coville, J. Organomet. Chem., 1994, $1,1$.

[3] B. Scheutz and R. Konrad. Business Briefing: Medical Device Manufacture and Technology, $\mathbf{2 0 0 5}, 39,1$.

[4] J.A. Langston, R.H. Colby, F. Shimizu, T. Suzuki, M. Aoki, and T. Chung, Macromol. Symp., 2008, 34 .

[5] J.A. Langston, R.H. Colby, T.M. Chung, F. Shimizu, T. Suzuki, and M. Aoki, Macromolecules, 2007, 40, 2712.

[6] A.H. Tullo, Chem. Eng. News, 2010, 88, 10.

[7] A. Gotsis, B. Zeevenhoven, and A. Hogt, Polym. Eng. Sci., 2004, 44, 973.

[8] H. Lau, S. Bhattacharya, and G. Field, Polymer Engineering \& Science 1994, 38, 1915.

[9] A. Lugao, H. Otaguro, D. Parra, A. Yoshiga, L. Lima, B. Artel, and S. Liberman, Radiat. Phys. Chem., 2007,76, 1688.

[10] D. Graebling, Macromolecules, 2002, 35, 4602.

[11] Y. Amintowlieh, C. Tzoganakis, and A. Penlidis, United States Patent Application No. 14/684,261, 2015.

[12] Y. Amintowlieh, C. Tzoganakis, and A. Penlidis, J Appl Polym Sci, 2014, 131, 41021.

[13] J. Tian, W. Yu, and C. Zhou, Polymer, 2006, 47, 7962.

[14] R. Shroff and H. Mavridis, J Appl Polym. Sci., 1995, 57, 1605.

[15] R. Shroff and H. Mavridis, Macromolecules, 1999, 32, 8454.

[16] Y. Amintowlieh, C. Tzoganakis, S. G. Hatzikiriakos and Alexander Penlidis, Polym. Degrad, Stab., 2014, 104, 1.

[17] M. Rätzsch, M. Arnold, E. Borsig, H. Bucka, and N. Reichelt, Prog. polym. Sci., 2002, 27, 1195-1282 (2002).

[18] J.S. Parent, A. Bodsworth, S.S. Sengupta, M. Kontopoulou, B.I. Chaudhary, D. Poche, and S. Cousteaux, Polymer, 2009, 50, 85.

[19] B. Wong and W. Baker, Polymer, 1997, 38, 2781.

[20] E. Borsig, M. Van Duin, A. Gotsis, and F. Picchioni, Eur. Polym. J., 2008, 44, 200.

[21] B. Kim and K. Kim, Adv. Polym. Technol., 2003,12, 263.

[22] Y. Amintowlieh, C. Tzoganakis, and A. Penlidis, Polym. Plast. Technol. \& Eng., 2015, DOI: $10.1080 / 03602559.2014 .986806$.

[23] G. He, Y. Huang, and Q. Yang, J. Wuhan Univ. of Technol.-Mater.Sci.Ed., 2013, $28,798$.

[24] G. He and C. Tzoganakis, Polym. Eng. \& Sci. 2011, 5l, 151.

[25] Y. Amintowlieh, C. Tzoganakis, and A. Penlidis, Polym. Eng. Sci., accepted, May 2015. 